\title{
Pathophysiology of Dyslipidemia in Cushing's Syndrome
}

\author{
Giorgio Arnaldi Valerio Mattia Scandali Laura Trementino Marina Cardinaletti \\ Gloria Appolloni Marco Boscaro
}

Division of Endocrinology, Department of Internal Medicine, Polytechnic University of Marche Region, Ancona, Italy

\section{Key Words}

Dyslipidemia • Free fatty acids · Steatosis · Insulin

resistance $\cdot$ AMP-activated protein kinase

\begin{abstract}
Dyslipidemia seems to be less frequent than other metabolic comorbidities in human Cushing's syndrome. Nevertheless, it plays an important role in determining the global cardiovascular risk in overt and subclinical Cushing's syndrome. In Cushing's syndrome, there is an increase of triglyceride and total cholesterol levels whereas HDL can be at variable levels. Overt and subclinical Cushing's syndrome share many features with metabolic syndrome including insulin resistance, abnormal fasting glucose levels, hypertension, obesity and dyslipidemia. The pathogenetic mechanisms are multifactorial, including direct and indirect cortisol action on lipolysis, free fatty acid production and turnover, verylow-density lipoprotein synthesis and fatty accumulation in the liver. AMP-activated protein kinase mediates many of glucocorticoid-induced metabolic changes. Insulin resistance plays a key role in determining lipid abnormalities. Other hormonal changes are involved including growth hormone, testosterone in men and estrogen in women, catecholamines and cytokines. In vitro, cortisol increases lipoprotein lipase in adipose tissues and particularly in visceral
\end{abstract}

fat where lipolysis is activated, resulting in the release of free fatty acids into the circulation. The increase of free fatty acids may enhance the accumulation of hepatic lipids reducing glucose uptake and activating various serine kinases which results in decreased insulin signaling. Moreover, mice with a liver-specific disruption of the glucocorticoid receptor had diminished hepatic triglycerides levels. In humans, a high prevalence (up to 20\%) of hepatic steatosis was also reported in patients with Cushing's syndrome. Genetic variations in the glucocorticoid receptors may also affect the activity of cortisol, lipid metabolism and cardiovascular risk.

Copyright $\odot 2010$ S. Karger AG, Basel

Glucocorticoids play a crucial role in energy homeostasis and they have complex and still not fully elucidated effects on lipid metabolism. Glucocorticoids modulate the expression of approximately $10 \%$ of human genes and in vitro a number of potential mechanisms through which glucocorticoids regulate lipid metabolism have been identified.

In vivo, it has been generally considered that chronic glucocorticoid excess is a secondary cause of dyslipidemia but the degree of lipid abnormalities in clinical conditions is quite variable and there are very few and conflicting studies on the prevalence of dyslipidemia in pa-

\section{KARGER}

Fax +41613061234 E-Mail karger@karger.ch www.karger.com

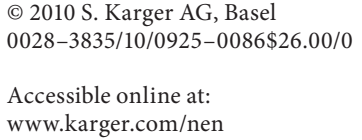

Giorgio Arnaldi

Clinica di Endocrinologia, Azienda Ospedaliero-Universitaria

Ospedali Riuniti di Ancona

IT-60100 Ancona (Italy)

Tel. +39 071887 061, Fax +39071 887 300, E-Mail arnaldi.giorgio@libero.it 
tients with exogenous and endogenous glucocorticoid excess $[1,2]$.

Several studies have suggested that long-term glucocorticoid therapy can cause dyslipidemia. However, a recent survey on 15,004 patients demonstrated that glucocorticoid use was not associated with an adverse lipid profile but with higher high-density lipoprotein (HDL) cholesterol among patients aged 60 years or older [1]. On the contrary, a clear glucocorticoid dose-response relation with BMI, triglyceride, total cholesterol, and lowdensity lipoprotein (LDL) levels was found in 1,707 hypopituitary patients on glucocorticoid replacement treatment [3].

Dyslipidemia is one of the features of human Cushing's syndrome including - in association with central obesity - hypertension, impaired glucose tolerance or diabetes, insulin resistance, hypercoagulability, and it determines an increased cardiovascular risk. In Cushing's syndrome, there is an increase in circulating very-lowdensity lipoprotein (VLDL) and LDL, but not high-density lipoprotein (HDL), with consequent elevation of triglycerides and total cholesterol levels [2]. These alterations normalize or improve after correction of hypercortisolism. In our series of 49 patients with Cushing's syndrome, total and LDL cholesterol were correlated with morning plasma cortisol and with cortisolemia after lowdose dexamethasone (Dex) suppression test. Interestingly, in contrast to other studies and in patients with metabolic syndrome (this condition shares many features with Cushing's syndrome), HDL-cholesterol levels were not reduced [4].

Subclinical hypercortisolism is also often associated with insulin resistance, abnormal fasting glucose levels, hypertension, obesity and dyslipidemia (high triglycerides and low HDL levels) [2].

This paper provides an update of the current knowledge on the pathophysiology of glucocorticoid-induced dyslipidemia where cellular and molecular mechanisms involved are multifactorial including direct and indirect cortisol-induced activity.

\section{Lipolytic Actions of Glucocorticoids}

Glucocorticoids regulate differentiation, function and distribution of adipose tissue. Glucocorticoids' effects on lipid metabolism in adipose tissue are controversial and may involve stimulation of both lipolysis and lipogenesis $[5,6]$. They can promote pre-adipocyte differentiation and inhibit adipose stromal cell proliferation, triggering a cascade of differentiation-dependent genes to facilitate adipogenesis. Glucocorticoids increase lipolysis in peripheral fat depots while promoting pre-adipocyte differentiation in central fat. Moreover, glucocorticoids may act synergistically with insulin to upregulate lipogenesis [5].

Recently, it has been demonstrated that glucocorticoids directly stimulate lipolysis in rat primary adipocytes in a dose- and time-dependent manner [7]. In this study, Dex stimulated the release of free fatty acids (FFA) and glycerol after $24 \mathrm{~h}$ of incubation. This action occurred even at a low Dex concentration; it was rapid starting at $4-8 \mathrm{~h}$ and increased continually to $32 \mathrm{~h}$. The findings that Dex increased intracellular cAMP levels and protein kinase A (PKA) activity and down-regulated cyclic-nucleotide phosphodiesterase $3 \mathrm{~B}$, the major enzyme responsible for cAMP hydrolysis, suggest that CAMP/ PKA system is functionally involved in the mechanism by which glucocorticoids stimulate lipolysis. Furthermore, incubation with Dex caused phosphorylation and downregulation of perilipin, a phosphoprotein that coats lipid droplets in adipocytes which regulates lipolysis. Phosphorylated perilipin changes conformation and, exposing the stored lipids, facilitates lipolysis [7].

Confirming previous studies, Xu et al. [7] showed that Dex significantly upregulated expression and promoted activity of hormone-sensitive lipase (HSL) and adipose triglycerides lipase, the two major lipases in adipocytes. However, Dex did not induce HSL translocation to the lipid droplets in differentiated adipocytes showing that Dex stimulation is weaker or different from typical PKA activation due to catecholamine.

That same study by Xu and coworkers [7] evaluated the in vivo lipolysis in epididymal adipose tissues isolated from male rats treated with Dex. In treated rats, Dex greatly increased FFA plasma concentration at 6 weeks and this was associated with increased lipase activity and lipolysis in adipose tissues.

In human adipocytes, Dex increased LPL mRNA and activity, and these effects were more marked in the omental adipose tissue, particularly in men. The maximum activity of LPL induced by insulin or insulin plus Dex was higher in the subcutaneous depot of women. In conclusion, there were differences between sexes and adipose depots studied [5].

Glucocorticoids may enhance lipolysis and modulate FFA mobilization through multiple mechanisms including a permissive effect, which has long been thought to be the principal one. In particular, glucocorticoids may modulate the dynamic responsiveness to other hormones 


\section{Chronic Glucocorticoid Excess}

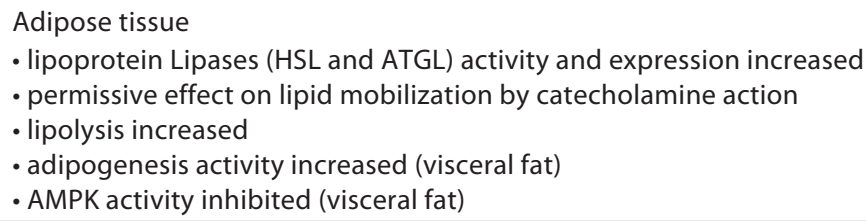

- lipoprotein Lipases (HSL and ATGL) activity and expression increased - permissive effect on lipid mobilization by catecholamine action - lipolysis increased

- adipogenesis activity increased (visceral fat)

- AMPK activity inhibited (visceral fat)

\section{Release of}

Free Fatty Acids (FFA) in portal circulation

Liver

- insulin resistance

- alteration of insulin signalling

(intracellular lipids phosphorylate serine sites on IRS-1)

- gluconeogenesis increased

- trialcyglycerides (TAG) storage and VLDL secretion increased

- de novo lipogenesis increased

- FFA $\beta$-oxidation inhibited

- AMPK activity increased

- hepatic steatosis
Fig. 1. Glucocorticoids' effect on adipose tissue and hepatic fatty acid metabolism. such as catecholamines and GH, thereby increasing their lipolytic action [6].

In vitro studies showed a combined lipolytic effect of $\mathrm{GH}$ and cortisol exceeding the GH-induced lipolysis in human subcutaneous abdominal adipose tissue. A recent study examined the effect of $\mathrm{GH}$, in both the absence and presence of Dex on lipolysis by omental adipose tissue explants from obese women in primary culture. An enhancement of lipolysis by GH in the presence of Dex but not by Dex or GH alone was observed. There was also a significant further stimulation by GH in the presence of Dex of hormone-sensitive lipase, perilipin, lipoprotein lipase and betal adrenergic receptor mRNA [8]. In a single-blinded, placebo-controlled, randomized in vivo study, GH and cortisol stimulate systemic and regional lipolysis independently and in an additive manner when coadministered in healthy men.

\section{Glucocorticoids Effects on Hepatic Fatty Acid Metabolism}

In humans, the effects of glucocorticoids on free fatty acid (FFA) are still controversial and the data available are not conclusive. In vivo studies showed that glucocorti- coids have either no effect or stimulate lipolysis, whereas others report an inhibiting effect of glucocorticoids on the lipolytic activity [6]. Moreover, in human studies these effects may be different in acute and chronic conditions. An excellent and recent article reviews this complex topic [6]. Briefly, in acute conditions such as stress, glucocorticoids increase whole body lipolysis, FFA uptake, spillover and turnover resulting in a 'hyperdynamic fatty acid system'. On the contrary, during recovery from stress, because of the fall of catecholamines and hyperinsulinemia, lypolysis decreases and triacylglycerides storage increases in the liver and visceral adipose tissue. This latter condition with absence of counter-regulatory hormones and insulin resistance is the condition observed in Cushing's syndrome.

The increase of FFA enhances the intracellular lipids stored in liver and muscle reducing glucose uptake, activating various serine kinases (JNK, IKK- $\beta$ ) which phosphorylate serine sites on insulin receptors, thus resulting in decreased insulin signaling pathway [reviewed in 9]. Thus, there is a causal link between hepatic fat accumulation and insulin resistance and this represents a further contribution to insulin resistance induced by glucocorticoid excess itself. Although these mechanisms have been demonstrated in vitro and in vivo in various animal 
models, available data in humans are still not conclusive and metabolic and molecular mechanisms of glucocorticoid-dependent fatty liver development and lipid metabolism remain largely unknown.

Noteworthy, a high prevalence of hepatic steatosis was also reported in patients treated with glucocorticoids and in patients with Cushing's syndrome where, in the latter, the presence of steatosis was significantly correlated with total abdominal and visceral fat [10].

Recently, a liver-specific disruption of the glucocorticoid receptor improved the steatosic phenotype in a fatty liver mouse model and normalized hepatic triglycerids levels [11]. This effect was mediated by glucocorticoid inhibition of hairy enhancer of split 1 (Hes1) gene expression which blocked hepatic de novo expression of pancreatic lipase. Glucocorticoids lead to hepatic steatosis compromising the fatty acid supply for $\beta$-oxidation and simultaneously inhibiting key enzyme expression and activity of $\beta$-oxidation (Acyl-CoA dehydrogenase, DH). In addition, insulin resistance plays a key role in determining lipid abnormalities and hepatic steatosis. In the liver, glucocorticoids cause hyperglycemia via the induction of gluconeogenic enzyme gene (PEPCK, G6Pase), increase VLDL production and secretion, enhance hepatic lipogenesis inducing key lipogenic enzyme activities (acetylCoA-carboxylase and fatty acid synthase) and inhibit fatty acid $\beta$-oxidation.

In addition, glucocorticoids increase apolipoprotein AIV (ApoAIV) expression during fasting, as demonstrated by the loss of ApoAIV fasting induction upon adrenalectomy and its restoration upon glucocorticoid replacement [12]. Moreover, glucocorticoids increase synthesis and secretion of apolipoprotein AI (ApoAI) by direct ApoAI promoter regulation via the glucocorticoid receptor [5].

\section{AMPK Mediates Many Glucocorticoid-Induced Metabolic Changes}

AMP-activated protein kinase (AMPK), playing a key role in regulating energy balance, is a 'guardian of cellular energy'. Once activated, it switches on catabolic pathways that produce ATP, such as glycolysis and fatty acid oxidation, while it switches off ATP-consuming anabolic pathways such as fatty acids, triglycerides and cholesterol synthesis. In particular, AMPK has been involved in the regulation of lipid metabolism [13].

Recently, in a rat model considered one of the best models of chronic glucocorticoid excess, AMPK was in- volved in many of the metabolic changes observed in Cushing's syndrome [14]. In this model, glucocorticoids changed AMPK activities in a tissue-specific manner explaining the increase in appetite, visceral obesity and dyslipidemia. In particular, glucocorticoids were shown to inhibit AMPK activity in visceral adipose tissue leading to increased lipogenesis and fat storage while they stimulated AMPK activity in liver. In addition, via HSL there is an increase of the release of lipids from adipose depots to the liver which may contribute to hepatic steatosis. In human adipocytes, Dex was shown to induce a fall in AMPK activity [14]. Patients with Cushing's syndrome exhibited a 70\% lower AMPK activity in visceral adipose tissue than control subjects with a consequent increase of fatty acid synthase expression. Of interest, the suppression of AMPK activity was proportional to the index of hypercortisolism [15].

\section{1ßHSD1 and Lipid Metabolism}

In humans, the $11 \beta$-hydroxysteroid dehydrogenase type 1 (11ßHSD1) catalyses the activation of cortisol from inactive cortisone thus it is a key regulator of cortisol action at cellular level [16].

Interestingly, in transgenic mice, overexpression of $11 \beta H S D 1$ in mature adipocytes was associated with the development of hepatic steatosis and the increase of total cholesterol and triglyceride levels [17]. In addition, hepatic overexpression of $11 \beta H S D 1$ caused mild insulin resistance, hepatic steatosis and increased hepatic lipid synthesis. The absence of obesity in this model confirmed an important role of glucocorticoid hormones in regulating lipid metabolism [18].

Furthermore, 11ßHSD1 knock-out animals displayed an improved lipid profile, an enhanced fatty acid oxidation, and a potentially atheroprotective phenotype [reviewed in 19]. In apolipoprotein E knockout mice, a specific 11ßHSD1 inhibitor was able to reduce the development of atherosclerosis by $84 \%$, although the mechanism for this phenomenon is not clear. In addition, it has been observed that treatment with $11 \beta H S D 1$ inhibitors improved triglyceridemia by reducing hepatic VLDL secretion and increased liver fatty acid oxidation. Recently, a 28-day treatment with INCB013739, a selective inhibitor of $11 \beta H S D 1$, improved insulin sensitivity and lowered total and LDL cholesterol in patients with type 2 diabetes mellitus [20]. 


\section{Conclusions}

Glucocorticoids seem to play a crucial role in the regulation of lipid metabolism even if their precise contribution is still unexplored and some data have yielded conflicting results. The pathogenetic mechanisms are multifactorial, including direct and indirect cortisol action on lipolysis, free fatty acid production and turnover, VLDL synthesis and fatty accumulation in liver. Insulin resistance plays a key role and AMPK mediates many glucocorticoid-induced metabolic changes. In addition, other hormonal changes are involved including $\mathrm{GH}$, testosterone, estrogens, catecholamines and cytokines. Finally, polymorphisms of the glucocorticoid receptor affecting cortisol sensitivity, lipid metabolism and cardiovascular risk, should also be taken into account [21].

\section{Disclosure Statement}

The authors have nothing to disclose.

\section{References}

1 Choi HK, Seeger JD: Glucocorticoid use and serum lipid levels in US adults: the Third National Health and Nutrition Examination Survey. Arthritis Rheum 2005;53:528-535.

-2 Arnaldi G, Angeli A, Atkinson AB, Bertagna X, Cavagnini F, Chrousos GP, Fava GA, Findling JW, Gaillard RC, Grossman AB, Kola B, Lacroix A, Mancini T, Mantero F, NewellPrice J, Nieman LK, Sonino N, Vance ML, Giustina A, Boscaro M: Diagnosis and complications of Cushing's syndrome: a consensus statement. J Clin Endocrinol Metab 2003; 88:5593-5602.

- 3 Filipsson $\mathrm{H}$, Monson JP, KoltowskaHäggström M, Mattsson A, Johannsson G: The impact of glucocorticoid replacement regimens on metabolic outcome and comorbidity in hypopituitary patients. J Clin Endocrinol Metab 2006;91:3954-3961.

4 Mancini T, Kola B, Mantero F, Boscaro M, Arnaldi G: High cardiovascular risk in patients with Cushing's syndrome according to 1999 WHO/ISH guidelines. Clin Endocrinol (Oxf) 2004;61:768-777.

$\checkmark 5$ Vegiopoulos A, Herzig S: Glucocorticoids, metabolism and metabolic diseases. Mol Cell Endocrinol 2007;275:43-61.

-6 Macfarlane DP, Forbes S, Walker BR: Glucocorticoids and fatty acid metabolism in humans: fuelling fat redistribution in the metabolic syndrome. J Endocrinol 2008; 197: 189-204.

7 Xu C, He J, Jiang H, Zu L, Zhai W, Pu S, Xu G: Direct effect of glucocorticoids on lipolysis in adipocytes. Mol Endocrinol 2009;23: 1161-1170.

-8 Fain JN, Cheema P, Tichansky DS, Madan AK: Stimulation of human omental adipose tissue lipolysis by growth hormone plus dexamethasone. Mol Cell Endocrinol 2008; 295:101-105.
9 Tilg H, Moschen AR: Insulin resistance, inflammation, and non-alcoholic fatty liver disease. Trends Endocrinol Metab 2008;19: 371-379.

10 Rockall AG, Sohaib SA, Evans D, Kaltsas G, Isidori AM, Monson JP, Besser GM, Grossman AB, Reznek RH: Hepatic steatosis in Cushing's syndrome: a radiological assessment using computed tomography. Eur J Endocrinol 2003; 149:543-548.

11 Lemke U, Krones-Herzig A, Berriel Diaz M, Narvekar P, Ziegler A, Vegiopoulos A, Cato AC, Bohl S, Klingmüller U, Screaton RA, Müller-Decker K, Kersten S, Herzig S: The glucocorticoid receptor controls hepatic dyslipidemia through Hes1. Cell Metab 2008; 8 : 212-223.

12 Hanniman EA, Lambert G, Inoue Y, Gonzalez FJ, Sinal CJ: Apolipoprotein A-IV is regulated by nutritional and metabolic stress: involvement of glucocorticoids, HNF-4 alpha, and PGC-1 alpha. J Lipid Res 2006;47:25032514.

13 Viollet B, Mounier R, Leclerc J, Yazigi A, Foretz M, Andreelli F: Targeting AMP-activated protein kinase as a novel therapeutic approach for the treatment of metabolic disorders. Diabetes Metab 2007;33:395-402.

14 Christ-Crain M, Kola B, Lolli F, Fekete C, Seboek D, Wittmann G, Feltrin D, Igreja SC, Ajodha S, Harvey-White J, Kunos G, Müller B, Pralong F, Aubert G, Arnaldi G, Giacchetti G, Boscaro M, Grossman AB, Korbonits M: AMP-activated protein kinase mediates glucocorticoid-induced metabolic changes: a novel mechanism in Cushing's syndrome. FASEB J 2008;22:1672-1683.
15 Kola B, Christ-Crain M, Lolli F, Arnaldi G, Giacchetti G, Boscaro M, Grossman AB, Korbonits M: Changes in adenosine $5^{\prime}$ monophosphate-activated protein kinase as a mechanism of visceral obesity in Cushing's syndrome. J Clin Endocrinol Metab 2008;93: 4969-4973.

16 Cooper MS, Stewart PM: 11Beta-hydroxysteroid dehydrogenase type 1 and its role in the hypothalamus-pituitary-adrenal axis, metabolic syndrome, and inflammation. J Clin Endocrinol Metab 2009;94:4645-4654.

17 Masuzaki H, Paterson J, Shinyama H, Morton NM, Mullins JJ, Seckl JR, Flier JS: A transgenic model of visceral obesity and the metabolic syndrome. Science 2001;294: 2166-2170.

18 Paterson JM, Morton NM, Fievet C, Kenyon CJ, Holmes MC, Staels B, Seckl JR, Mullins JJ: Metabolic syndrome without obesity: hepatic overexpression of 11beta-hydroxysteroid dehydrogenase type 1 in transgenic mice. Proc Natl Acad Sci USA 2004;101:7088-93.

19 Morton NM: Obesity and corticosteroids: 11beta-hydroxysteroid type 1 as a cause and therapeutic target in metabolic disease. Mol Cell Endocrinol 2010;316:154-164.

20 Rosenstock J, Banarer S, Fonseca V, Inzucchi S, Hollis G, Flores R, Levy R, Williams B, Huber R: Efficacy and safety of the 11-HSD1 inhibitor, INCB13739, added to metformin therapy in patients with type 2 diabetes. Proc 69th Sci Sessions Meet American Diabetes Association, New Orleans, 2009, p LB3, abstr 7-LB.

21 Manenschijn L, van den Akker EL, Lamberts SW, van Rossum EF: Clinical features associated with glucocorticoid receptor polymorphisms: an overview. Ann NY Acad Sci 2009; 1179:179-198. 